tal como sermón, ortodoxia, homilia, etc. No obstante, consideramos que hubiera sido práctico preservar junto a estos equivalentes la trans- cripción de la terminología árabe original para garantizar la máxima fluidez en los dos sentidos, o sea, para los traductores musulmanes que no manejan terminología cristiana ya que, aunque se trata de un manual para traductores del árabe al español también podría ser utilizado para traductores del español al árabe. Es importante que estos se familiaricen con la terminología cristiana para que puedan decidir cuándo un término (del o al árabe u otra lengua) requiere una equivalencia y cuándo una transcripción. Es un ejercicio, creemos, que puede ser doblemente provechoso. Dice Epalza (2004: 46) hablando precisamente de este punto: «la traducción del árabe al castellano requiere, por parte de los árabohablantes y también de los hispanohablantes, un conocimiento específico de la religión cristiana y de sus formas de expresión, muy marcadas en particular por la lengua latina, lengua oficial del cristianismo hispano».

En definitiva, estamos ante un libro de gran utilidad que lleva dignamente el título de manual ya que abunda en ejemplos muy prácticos que sirven como estrategia de trabajo no solo para la traducción de textos religiosos sino para cualquier otro tipo de textos. Serviría de igual modo como guía de la didáctica de traducción en estudios de postgrado dado que los textos expuestos en el manual, «escritos sean $\mathrm{u}$ orales», conllevan en sí una complejidad inmensa relativa, primero, a la estructura de la jutba y, luego, a las características de la lengua árabe y a la terminología y fórmulas de carácter religioso islámico. Esta complejidad requiere un manejo perfecto de la lengua árabe y española y un buen conocimiento de la sharía-ley islámica lo que, en muchas ocasiones, hace que sean textos difíciles de verter a otras lenguas.

\section{Traducción e identidad sexual: reescrituras audiovisuales desde la Teoría Queer}

ANTONIO JESÚS MARTÍNEZ PLEGUEZUELOS

Granada, Comares, 20I8, I40 págs.

\section{J. David González-Iglesias González} E1 libro Traducción e identidad sexual: reescrituras
Traducción e identidad sexual Reescrituras audiovisuales desde la Teoría Queer

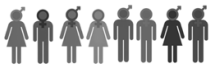

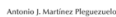
audiovisuales desde la Teoría Queer (Granada: Comares, 20I8), de Antonio Jesús Martínez Pleguezuelos, comienza con un prólogo de Rosario Martín Ruano en el que se presenta un nuevo acercamiento al binomio entre traducción e identidad. Esta dualidad, que ya ha sido estudiada por otros autores en el campo de los Estudios de Traducción, abre, no obstante, una nueva vía de análisis desde la perspectiva de las reescrituras que se llevan a cabo sobre las sexualidades en un mundo globalizado. Así, esta obra se centra en un aspecto que ha sido insuficientemente abordado hasta la fecha en un esfuerzo por ilustrar a académicos y profesionales acerca de los procedimientos de reescritura que existen en las traducciones transnacionales, particularmente en lo que respecta al tratamiento aplicado por los medios de comunicación.

A continuación, tras el prólogo, la introducción del libro establece las principales líneas sobre las que se desarrollarán los capítulos siguientes. Asimismo, se plantean en este apartado diferentes cuestiones que, desde una perspectiva traductológica, arrojarán luz sobre aspectos de gran relevancia para la traducción de textos de contenido y temática LGTB+. Gracias al enfoque multidisciplinar que se emplea, este primer apartado sienta las bases de un 
campo de investigación fértil y al mismo tiempo útil para académicos y profesionales.

A lo largo del primer capítulo, Martínez Pleguezuelos adopta un enfoque postestructuralista para analizar la naturaleza discursiva de las identidades y lleva a cabo un estudio minucioso desde las diferentes voces que han estudiado las formas en que el discurso construye las identidades desde ámbitos como los estudios culturales, los estudios LGTB+ o los estudios feministas. Así, el autor busca comprobar las consecuencias de nuestro uso del lenguaje a la hora de comunicar y a la hora de traducir cuando nos enfrentamos a temáticas relacionadas con la sexualidad y las sexualidades minoritarias con la ayuda de un soporte teórico sólido y riguroso sobre el que basar los capítulos posteriores. Es destacable el recurso a los postulados de Stuart Hall o Jonathan Potter para desgranar los diferentes procesos del discurso que construyen la realidad y la sexualidad. Por otra parte, el autor emplea en su revisión las premisas foucaultianas de la microfísica del poder y presenta con ellas un relato muy ilustrativo acerca de la aparición de la identidad del homosexual en los campos de la medicina y el derecho, así como la evolución de las identidades sexuales minoritarias a través del siglo xx y el xxi. Nos encontramos, sin duda, ante un texto de una sorprendente claridad, dada la profundidad de los temas que aborda, y de una vía de acceso privilegiada para comprender en toda su extensión los contenidos que se presentan posteriormente.

En el segundo capítulo el autor profundiza en el estudio de la construcción de la identidad sexual y recurre para ello a los postulados de Judith Butler sobre la performatividad, como continuación lógica de los principios de Foucault. Así, el texto coloca el foco sobre el concepto de identidad para cuestionar la posibilidad de que pueda llegar a considerarse un concepto estanco que defina al individuo de manera íntegra y unívoca, lo que supone el reconocimiento de categorías que superen las fronteras culturales y temporales. En palabras de Martínez Pleguezuelos, este postulado será particularmente significativo para los traductores, puesto que dichos mecanismos tienen la capacidad de dotar de carta de naturaleza a conceptos como «gay», «lesbiana» o «bisexual». Paradójicamente, en un acertado desarrollo de lo que otros autores como Cronin (2006) o Vidal Claramonte (2007) ya apuntaban, cualquier reescritura puede funcionar como una vía para romper los esquemas existentes sobre las identidades y reconocer así el amplio abanico de sexualidades que pueden encontrarse en diversos contextos. Cabe destacar una vez más en este punto la argumentación cartesiana del autor, que disecciona un tema de especial complejidad ante las necesidades actuales relativas a la representación no esencialista de otras identidades. Mediante estos postulados, el autor sienta las bases que, mediante los principios de la performatividad que presenta Butler, forjan la identidad de la persona y la propia naturaleza corporal de esta como superficie sobre la que se (re)inscribe el significado. Asimismo, se detalla la forma en que dichas líneas teóricas actuaron como cimientos para la edificación de una teoría queer en el corazón del colectivo LGTB + y para el reconocimiento de diferentes identidades que se hallaban (y aún hoy se hallan) en los márgenes de la sociedad debido a diferentes circunstancias culturales.

En su tercer capítulo, el libro presenta con una perspectiva innovadora la traducción como puente de unión y comunicación transnacional y herramienta para la globalización de las minorías sexuales. Como bien se apunta, son pocas las obras que abordan la globalización de la sexualidad, por lo que investigaciones como esta 
son necesarias para enfrentarnos a una cuestión que el mundo académico aún no ha analizado en detalle. Gracias al aparato teórico ensambla356 do en los capítulos precedentes, el texto estudia las identidades glocales que aparecen como resultado del contacto entre los discursos dominantes (globalizadores) y las escrituras locales que siguen representando un anclaje significativo para distintas comunidades. Mediante una perspectiva que integra los enfoques de los estudios de traducción, los estudios sobre globalización y los estudios LGTB+, el autor presenta diferentes ejemplos y argumentos sólidos que ilustran la forma en que los discursos occidentales se trasladan a todos los rincones del planeta y se infiltran en el sustrato social e ideológico de sus comunidades. Por último, se muestra cómo estas escrituras acaban por imponerse de manera solapada a los paradigmas identitarios preexistentes en estas comunidades en torno a la sexualidad.

Es en este punto en el que la traducción se presenta como un mecanismo idóneo para la transmisión de contenidos que responden a los intereses de determinados discursos dominantes dentro de los flujos de información intercultural. Los ejemplos que se presentan en el libro ilustran con sencillez el poder de los discursos traducidos que provienen de occidente y se exportan a distintas sociedades, así como las implicaciones directas que traen consigo y que afectan a la vida cotidiana de millones de personas. Martínez Pleguezuelos escenifica aquí cómo la cultura se utiliza como moneda de intercambio en un contexto global de imperialismo cultural que tiene un impacto notable en la aceptación y normalización de perfiles sexuales minoritarios, o en la ausencia de estas, y pone de relieve el hecho de que las escrituras hegemónicas sobre sexualidad no permiten, en muchas ocasiones, que dichos perfiles se visibi- licen. Destacan las referencias en este punto al contexto en diversos países de África o Asia y a la situación del colectivo LGTB+ en las periferias sociales de estos países. Con ellas, el autor subraya la importancia de la traducción como elemento subyacente en los procesos de configuración de identidades que se ven sometidos a sistemas externos de poder. De hecho, se da un paso más allá con una propuesta innovadora para interpretar la teoría queer en su conjunto como un fenómeno creado como resultado de la traducción de las teorías postestructuralistas de origen francés que se asimilaron en las universidades estadounidenses y del viaje de vuelta de estas al contexto europeo. En ellas, la reescritura y la incorporación de los postulados académicos son para el autor un terreno fértil en el que profundizar en el estudio de nuevas formas de percibir las sexualidades.

En la última parte, que reúne los dos capítulos finales, la obra presenta los ejemplos prácticos que se derivan de la propuesta teórica de las páginas anteriores. Mediante casos concretos, el cuarto capítulo lleva a cabo un análisis macrotextual en detalle de la representación tanto de personajes como de arcos argumentales LGTB+ en series de televisión estadounidenses con doblaje al castellano para un público español. Gracias a la perspectiva sólida que el autor conserva a lo largo del texto, resulta sencillo advertir en los ejemplos que las identidades distan de ser una idea inamovible en su concepto y a lo largo del tiempo, sino que permean otras culturas y lenguas. Los casos presentados, muy relevantes, atestiguan la naturaleza discursiva de la identidad y la forma en que al cruzar las fronteras lingüísticas necesitan la traducción como elemento fundamental para asentar nuevas estructuras identitarias en otras sociedades. Así, tal y como se puede ver en el quinto y último capítulo, el lenguaje se presenta como una herramienta de 
poder con la que es posible la creación de conocimiento. Por este motivo resulta especialmente útil el apartado dedicado al gayspeak y al camp talk desde una doble perspectiva traductora. Por una parte, se ofrece un abordaje académico que permite entender cuáles son los procesos discursivos utilizados en el seno de los propios colectivos LGTB + y la forma en que han ido evolucionando a lo largo del tiempo y a través del espacio gracias a un análisis diacrónico y diatópico. Por otra parte, y ya desde un prisma más práctico y profesional, se analizan estas realidades como valioso recurso que los traductores pueden emplear en la solución de problemas léxicos o semánticos cuando se enfrentan a un texto marcado por estos rasgos concretos. Por último, el autor presenta distintos ejemplos extraídos de series televisivas de temática LGTB+ desde los años noventa hasta la actualidad para ilustrar la forma en que han evolucionado los procesos y mecanismos de reescritura, lo que reafirma los planteamientos esgrimidos en la obra en torno a la naturaleza discursiva de la sexualidad. El análisis compara las versiones original y doblada de series como Will E Grace, Queer as Folk, Transparent o Looking, y su enfoque desde las últimas tendencias de los estudios de traducción audiovisual reiteran la importancia de esta investigación en el ámbito de la traducción.

Es preciso concluir añadiendo que Traducción e identidad sexual: reescrituras audiovisuales desde la Teoría Queer es una obra necesaria y reveladora que abre nuevas vías de investigación en el campo académico que resultarán sin duda muy fructíferas, pero que sirve al mismo tiempo como eficaz guía para los traductores profesionales que deseen incorporar una perspectiva integradora en el ejercicio de su labor.

\section{El viaje de la literatura. Aportaciones a una didáctica de la traducción literaria} CARLOS FORTEA (COORD.)

Cátedra, Madrid, 2018, 2015 págs.

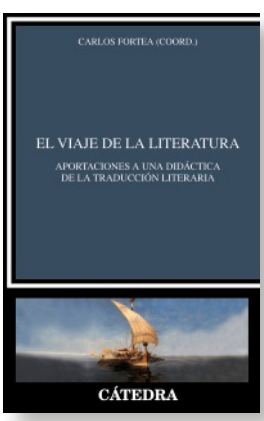

Carmen Clavero Fernández Creatividad y aprendizaje, un binomio inseparable para iniciar y culminar con éxito el viaje de ida y vuelta de la traducción literaria. Esta es la reflexión que, en su particular singladura a través de once capítulos, nos quieren transmitir los autores de este libro.

El viaje de la literatura se publicó un año antes del quingentésimo aniversario del viaje de Magallanes y Elcano. En su empeño por circunnavegar la tierra, ambos marineros también combinaron su ingenio y creatividad con enormes dosis de experiencia para conducir cinco naves y algo más de doscientos hombres a través de los océanos, en un viaje de ida y vuelta relatado por el italiano Pigaffeta, que convivió durante los tres años de la expedición con tripulantes españoles, portugueses, italianos $\mathrm{u}$ holandeses. La presencia de varias lenguas a bordo no impidió culminar semejante hazaña.

Pero volvamos a nuestro asunto: el libro publicado por el grupo de investigación TradLit: Aproximación a una teoría de la traducción literaria a través de su didáctica, creado en 2010 por profesores de la Facultad de Traducción y Documentación de la Universidad de Salamanca. A son de mar y con las velas aparejadas, iremos abarloando cada capítulo de esta obra que, partiendo del Marco de referencia PETRA-E para la educación y la formación de traductores literarios (2016), aspira a ser una guía para estudiantes y profesores interesados en la traducción literaria 